\title{
A Revised Magnitude and Distance Amplitude Correction (MDAC2) Procedure for Regional Seismic Discriminants: Theory and Testing at NTS
}

W.R. Walter, S.R. Taylor

December 21, 2001

U.S. Department of Energy

Lawrence Livermore

National

Laboratory

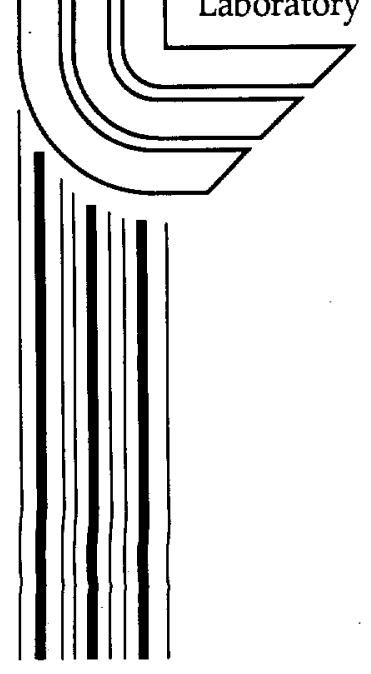




\section{DISCLAIMER}

This document was prepared as an account of work sponsored by an agency of the United States Government. Neither the United States Government nor the University of California nor any of their employees, makes any warranty, express or implied, or assumes any legal liability or responsibility for the accuracy, completeness, or usefulness of any information, apparatus, product, or process disclosed, or represents that its use would not infringe privately owned rights. Reference herein to any specific commercial product, process, or service by trade name, trademark, manufacturer, or otherwise, does not necessarily constitute or imply its endorsement, recommendation, or favoring by the United States Government or the University of California. The views and opinions of authors expressed herein do not necessarily state or reflect those of the United States Government or the University of California, and shall not be used for advertising or product endorsement purposes.

This work was performed under the auspices of the U.S. Department of Energy by the University of California, Lawrence Livermore National Laboratory under Contract No. W-7405-Eng-48.

This report has been reproduced directly from the best available copy.

Available electronically at http://www.doe.gov/bridge

Available for a processing fee to U.S. Department of Energy

and its contractors in paper from

U.S. Department of Energy

Office of Scientific and Technical Information

P.O. Box 62

Oak Ridge, TN 37831-0062

Telephone: (865) 576-8401

Facsimile: (865) 576-5728

E-mail: reports@adonis.osti.gov

Available for the sale to the public from

U.S. Department of Commerce

National Technical Information Service

5285 Port Royal Road

Springfield, VA 22161

Telephone: (800) 553-6847

Facsimile: (703) 605-6900

E-mail: orders@ntis.fedworld.gov

Online ordering: http://www.ntis.gov/ordering.htm

OR

Lawrence Livermore National Laboratory

Technical Information Department's Digital Library

http://www.llnl.gov/tid/Library.html 
A Revised Magnitude and Distance Amplitude Correction (MDAC2) Procedure for Regional Seismic Discriminants: Theory and Testing at NTS

W. R. Walter and S. R. Taylor*

Lawrence Livermore National Laboratory

*Los Alamos National Laboratory

December 21, 2001

UCRL-ID-XXXXXX

LA-UR-01-XXXX 


\begin{abstract}
The Magnitude and Distance Amplitude Correction (MDAC; Taylor and Hartse, 1998; Taylor et al., 2002) procedure for correcting regional seismic amplitudes for seismic event identification has been modified to include more realistic earthquake source models and source scaling. In the MDAC2 formulation we generalize the Brune (1970) earthquake source spectrum to use a more physical apparent stress model that can represent non-constant stress-drop scaling. We also event include a parameter that allows for variable $\mathrm{P}$-wave and $\mathrm{S}$-wave corner frequency scaling, imposing some of the constraints of ratio correction techniques (Rodger and Walter, 2002). Very stable moment magnitude measures (Mayeda et al., 2002) from regional coda wave envelopes that have been tied to independently derived regional seismic moments are incorporated. This eliminates two fitting parameters that were necessary in relating seismic moment to magnitude. The incorporation of Bayesian tomography to replace the assumption of a constant $\mathrm{Q}_{0}$ model is also described. These modifications allow for more flexibility in the MDAC grid-search procedure. The direct tie to regional seismic moment rather than body wave magnitude reduces effects of upper mantle bias on the corrected amplitudes. In this paper, we develop the theory and test the formulation on Nevada Test Site (NTS) data.
\end{abstract}




\section{Introduction}

Effective earthquake-explosion seismic discrimination has been demonstrated in a broad variety of studies using ratios of regional amplitudes in high frequency (primarily 1-20 $\mathrm{Hz}$ ) bands. When the earthquake and explosion are nearly co-located and have similar size we can understand the observed seismic contrast in the relative P-to-Lg excitation shown Figure 1 in terms of depth, material property, focal mechanism and source time function differences. The availability of reference earthquakes and particularly nuclear tests to compare to a new event in question however is highly non-uniform and limited. Therefore in real monitoring situations we are often interested in comparing events that are not co-located and may have quite different sizes. In order to make sure any observed differences between a new event in question and the reference events (or models) are not due to differences in path or magnitude we must correct for these effects.

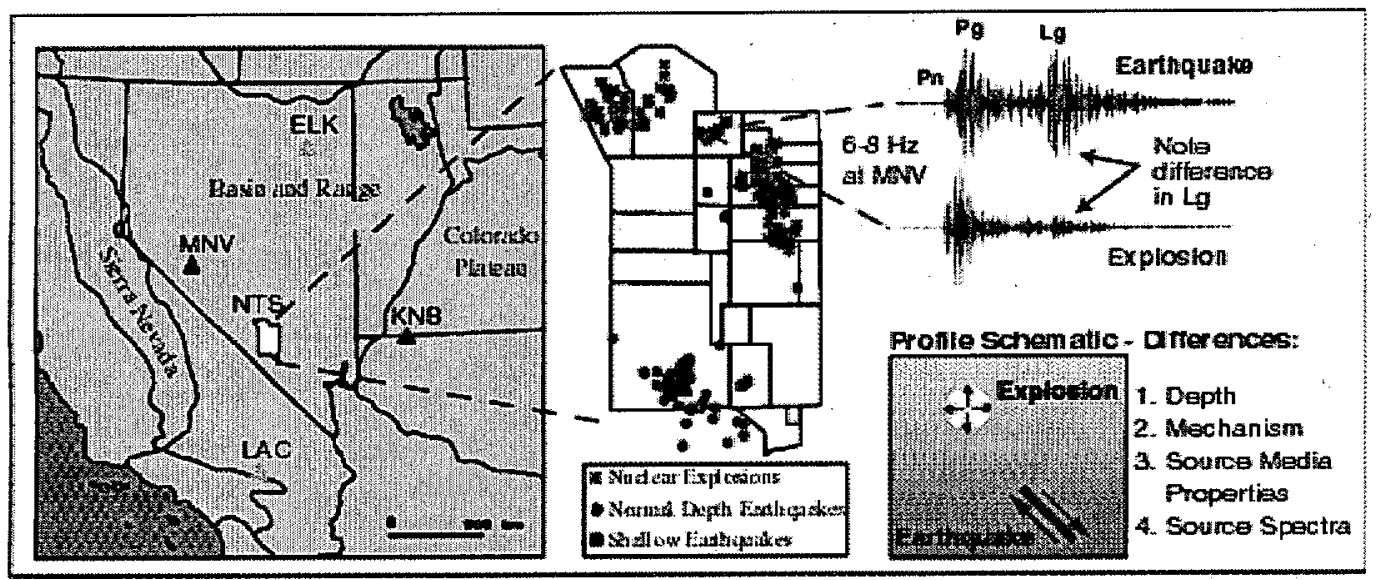

Figure 1. Overview of earthquake-explosion discrimination at the Nevada Test Site (NTS). Left-hand map shows the location of NTS and some regional seismic stations. Center plot shows NTS earthquakes and explosions studies by Walter $e t$ al. (1995). Right-hand side shows high frequency seismograms at station KNB demonstrating the characteristic difference in relative $\mathrm{P}(\mathrm{Pn}, \mathrm{Pg})$ amplitude to $\mathrm{Lg}$ amplitude. The causes of this difference our outlined in the schematic profile.

The goal of the original Magnitude and Distance Amplitude Correction (MDAC) procedure (Taylor and Hartse, 1998; Taylor et al., 2002) was to remove any magnitude and distance trends in the regional phase amplitudes and any of the discriminants formed from those amplitudes. The corrected amplitudes can then be taken in combination to form multivariate normal discriminants. Because the entire spectrum of each phase is corrected this technique allows for maximal flexibility in the construction of optimum discriminants. MDAC enables the construction of discriminants that follow a multivariate normal distribution. This allows for the application of likelihood-based classification methods having a sound statistical basis.

Using a large set of earthquakes, the MDAC methodology involves the fitting of a simple one-dimensional propagation model and earthquake source model to observed regional seismic spectra. The method generates synthetic spectra which when properly calibrated and removed from the observations can eliminate magnitude and distance correlations. 
Spatial corrections to the MDAC residuals (corrections) can be computed using nonstationary Bayesian kriging (Schultz et al., 1998) to account for departures from the simple one-dimensional propagation model. In this report, we will describe the MDAC2 methodology and then present some applications to NTS earthquake and explosion data.

\section{MDAC2 Methodology}

To model and understand regional seismic spectra we need to break down the observations into their component parts. The observed instrument-corrected regional phase spectra can be thought of as a convolution between the recording instrument, the source and the path. In the frequency domain we can write this out as a cascading multiplication:

$$
A_{o}(\omega, R)=S(\omega) G(R) P(\omega) B(\omega, R)
$$

where $\omega$ is the angular frequency, $R$ is distance and:

$S(\omega)$ is the source spectrum, $G(R)$ is the geometrical spreading,

$P(\omega)$ is the frequency dependent site effect $B(\omega, R)$ is the apparent attenuation

$c$ is the velocity of the phase and $Q(\omega)$ is the frequency dependent quality factor. Here we have split the path effect into three pieces: 1) a frequency independent geometrical spreading component, 2) a range independent and frequency dependent site effect component, and 3) an apparent attenuation component.

The MDAC2 prediction of the observed, instrument-corrected spectra is then given by:

$$
A_{m}(\omega, R)=S(\omega) G(R) P(\omega) B(\omega, R)
$$

We linearize equation (2) by taking the logarithm of both sides:

$$
\log A_{m}(\omega, R)=\log S(\omega)+\log G(R)+\log P(\omega)+\log B(\omega, R)
$$

To remove the data trends with distance and magnitude, we correct the observed spectrum, $A_{c}(\omega, R)$, by subtracting the $\log$ of the MDAC2 spectrum in equation (3):

$$
\log A_{\mathrm{c}}(\omega, R)=\log A_{o}(\omega, R)-\log A_{m}(\omega, R)
$$

These corrected spectra or residuals can then be kriged to further reduce unmodeled path effects (e.g. Schultz et al., 1998). In order to use equation (3) to make these corrections we must define each of its terms. Here we will discuss each one below.

\section{Source Spectra S( $\omega)$}

The original MDAC procedure involved estimating and removing a simple theoretical earthquake spectrum from the data to remove any magnitude and distance trends in the regional phase amplitudes and any discriminants formed from those amplitudes (Taylor and Hartse, 1998; Taylor et al., 1999). Here we refine and improve the procedure by 
generalizing the source model, taking advantage of independent moment estimates and reducing some of the free parameters.

The source spectrum depends upon the seismic moment and stress drop and can have additional complications due to non-constant stress drop scaling and differential P/S corner frequency effects that we will discuss later. We require different phases for the same event recorded at the same station to have the same moment and apparent stress (or stress drop) values and other source parameters, such as corner frequencies, to be related to each other. This effectively imposes some of the ratio constraints discussed in Rodgers and Walter (2002) on the amplitudes and improves discrimination performance. While such models of source spectra are certainly oversimplified, they have proven track records of providing good first-order fits to real earthquakes. In addition they also provide simple theoretical models to use in aseismic areas.

We revise the original MDAC formulation in two ways:

1) We take advantage of the very stable moment magnitude determinations from regional coda envelopes (see Mayeda et al., 2002). The coda-based technique provides an accurate and independent estimate of moment from as few as one station. Using a direct measure of moment also eliminates the need to scale from other magnitudes such as $m_{b}$ to. moment eliminating two fitting parameters from the original formulation (Taylor $e t a l$., 2002). The combination of using the more stable coda measure and eliminating two free parameters should reduce scatter in the resulting correction.

2) We generalize the Brune (1970) source spectrum to allow for arbitrary stress scaling. A problem with non-constant stress drop scaling is that the Brune (1970) model is specifically set up as constant stress drop model. Taylor et al. (2002) implemented a non-constant scaling, but at a cost of having to introduce a non-physical parameter whose units varied with scaling. This was necessary in order to maintain proper units for stress drop (MPa).

We reformulate the source term as follows, starting with the Brune (1970) spectral shape:

$$
S(\omega)=\frac{S_{0}}{1+\left(\frac{\omega}{\omega_{c}}\right)^{2}}
$$

where $S_{o}$ is the zero frequency spectral level and $\omega_{c}$ is the angular corner frequency $\left(2 \pi f_{c}\right)$. We parameterize $S_{o}$ using the seismic moment, $M_{o}$ and the well known relationship between them (e.g. Aki and Richards, 1980):

$$
S_{o}=F M_{o} \quad \text { where } \quad F=\frac{R_{0 \phi}}{4 \pi\left(\rho_{s} \rho_{r} c_{s}^{5} c_{r}\right)^{1 / 2}}
$$

where $R_{\theta \phi}$ is the radiation pattern coefficient appropriate to the given wave type, $\rho$ is the density, $c$ is the velocity of the given wave type and the subscripts $r$ and $s$ refer to the receiver and source region respectively. Note we have already removed the geometrical spreading term $G(R)$ as a separate function in equation (3). To parameterize the corner 
frequency, we reformulate the stress drop in terms of a more general stress parameter, the apparent stress (e.g. Wyss, 1970):

$$
\sigma_{a}=\frac{\mu E}{M_{o}}
$$

where $E$ is the seismically radiated energy and $\mu$ is the rigidity at the source. The seismically radiated energy in any particular wave type with velocity $c$ and a spectrum given by equation (5) can be determined by integrating to energy flux through an imaginary sphere of radius $R$ around the source and considering only source properties we find (Walter and Brune, 1993):

$$
E_{c}=\pi \rho, R^{2} c_{s} S_{o}^{2} \omega_{c}^{3}
$$

substituting in equation (6) to rewrite $S_{o}$ in terms of $M_{o}$ and considering the material property values at radius $\mathrm{R}$ to be those of the source, and geometrical spreading to be $1 / R$ gives:

$$
E_{c}=\frac{R_{\phi}^{2} M_{\mathrm{o}}^{2} \omega_{\mathrm{c}}^{3}}{16 \pi \rho_{s} c_{s}^{s}}
$$

Equation (9) can now be used with equation (7) to determine the corner frequency scaling for a particular phase in terms of apparent stress.

To keep the source parameters related we want to use a single apparent stress parameter for each event and therefore we sum the energy from all the regional phases (e.g. Pn, Pg, $\mathrm{Sn}$ and $\mathrm{Lg}$ ) to find the total radiated body-wave energy to put into equation (7). This effectively imposes some of the constraints that have been used in ratio correction techniques (e.g. Rodgers and Walter, 2002) while retaining the flexibility of working with individual corrected phase amplitudes. For simplicity we group the P-waves and Swaves together and use an additional parameter to link the $\mathrm{P}$ - and $\mathrm{S}$ wave corner frequencies:

$$
\omega_{c p}=\xi \omega_{c s}
$$

where subscripts $P$ and $S$ denote $\mathrm{P}$ - and $\mathrm{S}$-wave corner frequencies and $\zeta$ is the scale factor. For the Brune (1970) model $\zeta$ is the ratio of the wave velocities, but other studies have shown that the corner frequencies are more equal $(\zeta=1)$. So typically we set $1 \leq \varsigma \leq v_{p} / v_{s}$. By including this parameter we allow maximum flexibility. We treat the energy radiated as the simple sum of $P$ and $S$ wave energy:

$$
E=E_{p}+E_{s}=\frac{M_{s}^{2}}{16 \pi \rho_{s}}\left(\frac{R_{Q p}^{2} w_{s p}^{3}}{\alpha_{s}^{5}}+\frac{R_{\alpha s}^{2} w_{s s}^{3}}{\beta_{s}^{5}}\right)
$$

and use it along with equations (7), (9) and (10) to solve for the S-wave corner frequency: 


$$
\omega_{c s}=\left(\frac{K \sigma_{a}}{M_{o}}\right)^{1 / 3} \quad \text { where } \quad K=\frac{16 \pi}{\beta_{s}^{2}\left(\frac{R_{\phi_{\alpha}}{ }^{2} \zeta^{3}}{\alpha_{s}^{5}}+\frac{R_{\phi \sigma}{ }^{2}}{\beta_{s}^{5}}\right)}
$$

Here we also used $\mu=\rho_{s} \beta_{s}{ }^{2}$. Equation (12) provides a general relationship between corner frequency, moment and apparent stress. A number of previous studies (e.g.

Abercrombie, 1995, Mayeda and Walter, 1996) have shown that apparent stress can increase with increasing moment. In order to allow this possibility we introduce a generalized apparent stress moment relationship:

$$
\sigma_{a}=\sigma_{a}\left(\frac{M_{o}}{M_{o}}\right)^{\psi}
$$

where $\sigma_{a}{ }^{\prime}$ is the apparent stress at the reference moment $M_{o}{ }^{\prime}$. For constant apparent stress $\psi=0$ and the corner frequency in equation (12) scales as $M_{o}^{-1 / 3}$ as in the Brune (1970) model. This formulation using equations (12) and (13) allows us to put in a physical nonconstant stress scaling such as the quarter root scaling $(\psi=1 / 4)$ observed by Mayeda and Walter (1996) for western U.S. earthquakes. This also allows for better agreement between the coda-determined spectra and those produced by MDAC2.

Geometrical Spreading: $G(R)$

Seismic waves spread out as they propagate through the Earth. Under conservation of energy this leads to amplitudes diminishing as well. Traditionally this geometrical spreading effect has been characterized as proportional to distance to a negative power. We follow Street et al. (1975) to define a geometrical spreading factor that has a critical distance $R_{o}$ within which the spreading is spherical and beyond which is decays as distance to the power $\eta$.

$$
G(R)=\left(\begin{array}{c}
\frac{1}{R} \text { where } \quad R<R_{o} \\
\frac{1}{R_{o}}\left(\frac{R_{o}}{R}\right)^{n} \text { where } \quad R \geq R_{o}
\end{array}\right)
$$

This formulation accounts for the crustal waveguide effect on some phases such as $\mathrm{Pg}$ and Lg. For upper mantle phases $\mathrm{Pn}$ and $\mathrm{Sn}$ we can set $R_{o}$ to a small number (e.g. $1 \mathrm{~km}$ ). This term has not changed from the original Taylor et al (2002) formulation. We recognize that complex velocity structure can make the geometrical spreading factor frequency dependent for upper mantle phases (e.g. Sereno and Given, 1990) and anticipate that station-centric kriging will aid in accounting for some of these complexities.

Site effect: $P(\omega)$

It is well known that even for small changes in recording location (such as elements within an array) the amplitude of an observed seismic wave can vary significantly. Part of this is due to impedance effects, that is under conservation of energy the amplitude of the seismic wave will increase in slower, less dense material (e.g. sediment) and decrease in faster more dense material (hard rock). In addition there can be resonance due to 
layering, topography and scattering effects that cause small-scale variations in amplitudes.

We allow for a frequency dependent site effect as $P(\omega)$ independent of range similar to that cause by impedance contrasts. Site effects that are functions of range, azimuth or location are accounted for in other terms or in the kriging of the residuals. The site effect term can also account for unmodeled relative source excitation factors between phases so in some sense combines site and relative phase excitation terms.

Apparent Attenuation: $B(\omega, R)$

As waves propagate through the imperfectly elastic earth they lose energy and their amplitude decreases. This can be caused both by inelastic energy absorption and by scattering. In addition it can be difficult to separate these attenuation effects from geometrical spreading effects. Here we do not try to separate intrinsic and scattering attenuation. Rather for a given geometrical spreading we model the remaining effect as apparent attenuation defined in terms of the Quality or Q factor:

$$
B(\omega, R)=\exp \left(-\frac{\omega R}{2 Q(\omega) c}\right)
$$

We follow the standard practice for regional phases of allowing a linear frequency dependent $Q$ in the form:

$$
Q(\omega)=Q_{o} f^{\gamma}=Q_{o}\left(\frac{\omega}{2 \pi}\right)^{\gamma}
$$

If previous studies or $\mathrm{Q}$ tomography is available independently then these can be used to determine the attenuation. Otherwise the $Q_{0}$ and $\gamma$ terms are solved for. This apparent attenuation formulation is the same as in the original MDAC (Taylor and Hartse, 1998; Taylor et al., 2002).

We have added the ability to incorporate Bayesian attenuation tomography to the MDAC2 methodology (Tarantola, 1987; Taylor et al., 2001). Tomographic results from prior studies can be used or built upon using a Bayesian approach that accounts for inadequacies of the assumption of a constant $Q_{0}$ for a large region. The advantages of a Bayesian approach to tomography are that large-scale and high-resolution tomographic models available from other well-accepted studies can be used as prior background models. The resulting refined tomographic model blends into these prior background models. Moreover, the error budget is well established in a Bayesian framework. We assume a general linear Gaussian (least squares) model, where the covariance matrix is partitioned into data and prior model components. Uncertain data are naturally down weighted and certain model components will be subject to small perturbations.

\section{$M D A C 2$}

Now that we have defined all the terms in equation (3) we substitute them in and write out the predicted MDAC2 log spectrum: 


$$
\log A_{m}(\omega, R)=\log \left(F M_{o}\right)-\log \left(1+\left(\frac{\omega}{\omega_{c}}\right)^{2}\right)+\log G(R)+P(f)-\frac{\pi R \log e}{\ell_{o} c}\left(\frac{\omega}{2 \pi}\right)^{(1-r)} \log (e)(17)
$$

for the appropriate wave type with velocity $c$. So for a given moment $M_{o}$ and distance $R$, the MDAC2 spectrum is calculated using equation (17), $F$ is determined from equation $(6), \omega_{c}$ from equations (12) and (13), and $G(R)$ is given by equation (14).

Application to NTS events

We first want to test and compare the MDAC2 spectral predictions to data in a region with well-known attenuation and geometrical spreading to make sure everything is working properly. A natural choice for initial testing are the well studied NTS regional data recorded at LLNL's regional stations in the Basin and Range region of the Western U.S. The June 1992 Little Skull Mountain earthquake occurred in the southern portion of NTS (see Figure 1) and was followed by many aftershocks. These data have been well studied for discrimination (Walter et al., 1995) and source parameters and moment magnitudes were calculated for them (Mayeda and Walter, 1996).

Attenuation and geometrical spreading values for regional phases have been the subject of many studies in the Basin and Range (e.g. Chavez and Priestley, 1986, McCormack et al, 1994, Benz et al 1997). We use geometrical spreading and $Q$ values of Walter (unpublished data) which are very similar to those found in prior studies. They are shown in the table below:

\begin{tabular}{|l|l|l|l|l|}
\hline Phase & $\mathrm{R}_{0}$ & $\eta$ & $\mathrm{Q}_{\mathrm{o}}$ & $\gamma$ \\
\hline $\mathrm{Pn}$ & 1 & 1.1 & 210 & .65 \\
\hline $\mathrm{Pg}$ & 100. & 0.5 & 190 & .45 \\
\hline $\mathrm{Lg}$ & 100. & 0.5 & 200 & .54 \\
\hline
\end{tabular}

An example of the MDAC2 simultaneous fit to Pn, $\mathrm{Pg}$ and $\mathrm{Lg}$ spectra is shown in Figure 2 for the Little Skull Mountain earthquake mainshock recorded at station KNB in Utah about $300 \mathrm{~km}$ distant (see Figure 1). Figure 2 shows the effect on the spectral fitting of the variable $\zeta$, the $\mathrm{P}$ to $\mathrm{S}$ corner frequency ratio. The left-hand plot is for equal corner frequency values while the right hand plot is for high $\mathrm{P}$ corner frequency by the ratio of the wave velocities (1.73). The best fit appears to be closer to equal corner frequency values. 
MDAC2 fit to Mw=5.68 Little Skull Mountain earthquake for two different PIS corner frequency values
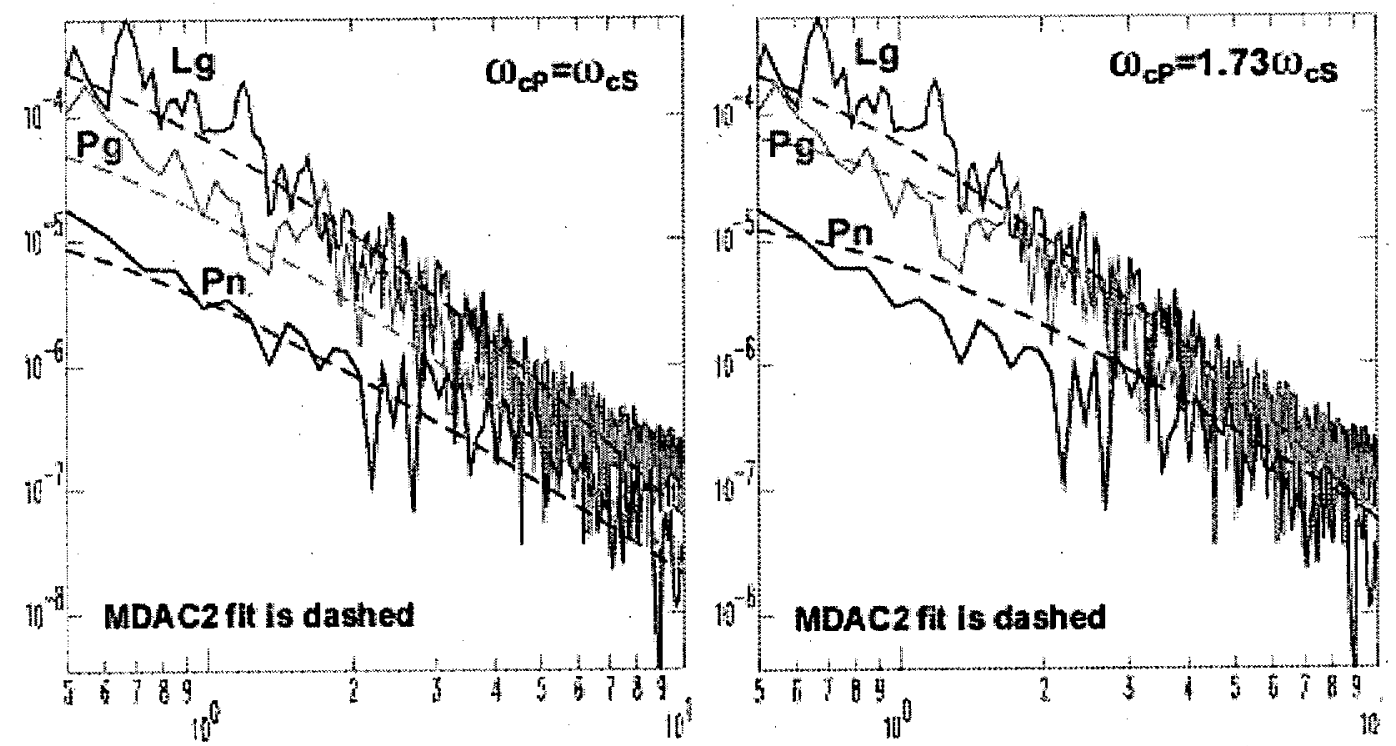

Figure 2. Comparison of the effect of P-to-S corner frequency variable on spectral fits.

Another example of MDAC2 scaling is shown in Figure 3. Here we show Lg spectra for the Little Skull Mountain mainshock and two aftershocks. The coda moment magnitudes are from a previous study (Mayeda and Walter, 1996). Note that the constant apparent stress model on the left does not fit the data as well as the stress scaling with $\mathrm{M}_{\mathrm{o}}{ }^{1 / 4}$ shown on the right. This is consistent with our previous spectral studies using coda (Mayeda and Walter, 1996). 


\section{An example of MDAC2 fits to Lg spectra from the 1992 Little Skull Mountain sequence at the Nevada Test Site: Mainshock and two aftershocks.}
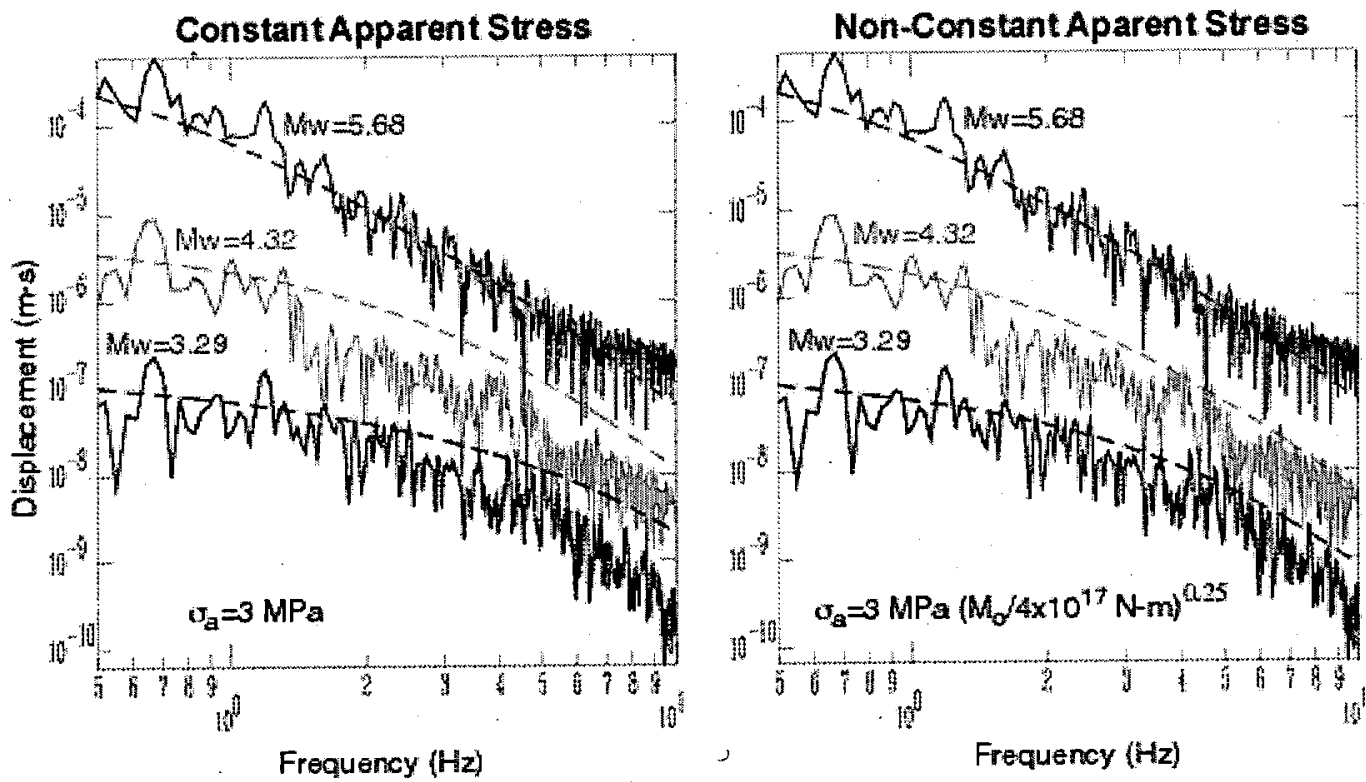

Figure 3. Comparison of the effect of apparent stress scaling on the spectral fits.

\section{Application to Uncalibrated Regions}

For uncalibrated regions the geometrical spreading parameters $\left(R_{0}, \eta\right)$, attenuation parameters $\left(Q_{0}, \gamma\right)$ and source scaling parameters need to be solved for. As with previous versions of spectral (Taylor et al, 2002) and ratio (Rodgers and Walter, 2002) fitting we use a grid search technique. In practice we search only a few values of $\zeta$ (e.g. 1, 1.73) and $\psi$ (e.g. 0 and 0.25 ) and concentrate our search on the other parameters. The MDAC 2 formulation has been coded in Matlab by Steve Taylor and in FORTRAN by Arthur Rodgers. Tests of the FORTRAN code show that fitting several hundred events at a given station is quit feasible in a reasonable amount of time. The full description of the grid search codes, their application to uncalibrated regions and the resulting discrimination performance is beyond the scope of this paper and will be included in a future more comprehensive write up.

\section{Acknowledgments}

We wish to thank Arthur Rodgers for comments which improved the manuscript. This work is performed under the auspices of the U.S. Department of Energy by Lawrence Livermore National Laboratory under contract W-7405-ENG-48 and Los Alamos National Laboratory under contract W-7405-ENG-36. 


\section{References}

Aki, K. and P. G. Richards, (1980). Quantitative Seismology, W. H. Freeman, New York.

Benz, H. M., A. Frankel, and D. M. Boore, (1997). Regional Lg attenuation in the continental United States, Bull. Seism. Soc. Am., 87, 606-619.

Brune, J., (1970). Tectonic stress and the spectra from seismic shear waves earthquakes, J. Geophys. Res., 75, 4997-5009.

Chavez, D., and K. F. Priestley (1986). Measurement of frequency dependent Lg attenuation in the Great Basin, Geophys. Res. Lett., 13, 551-554.

Mayeda, K. M. and W. R. Walter, (1996). Moment, energy, stress drop and source spectra of Western U.S. earthquakes from regional coda envelopes, J. Geophys. Res., $101,11,195-11,208$.

Mayeda, K., A. Hofstetter, J. L. O'Boyle, and W. R. Walter, (2002). Stable and transportable regional magnitudes based on coda-derived moment-rate spectra, submitted to Bull. Seis. Soc. Am.

McCormack, D. A., K. F. Priestley, and H. J. Patton, (1994). Distance effects on regional discriminants along a seismic profile in Northwest Nevada; NPE and nuclear results, in Proceedings of the Symposium on the Non-proliferation Experiment, edited by M. Denny, Lawrence Livermore National Laboratory, CONF 9404100, pages 6-254 to 6-271.

Rodgers, A. J. and W. R. Walter, (2000). Seismic Discrimination of the May 11, 1998 Indian Nuclear Test with Short-Period Regional Data From Station NIL (Nilore, Pakistan), Pure Appl. Geophys. (in press).

Schultz, C, S. Myers, J. Hipp, and C. Young (1998). Nonstationary Bayesian kriging: a predictive technique to generate corrections for detection, location and discrimination, Bull. Seism. Soc. Am., 88 1275-1288.

Sereno, T.J. and J.W. Given (1990). Pn attenuation for a spherically symmetric earth model, Geophys. Res. Lett., 17, 1141-1144.

Stevens, J. L. and S. M. Day (1985). The physical basis of mb:Ms and variable frequency magnitude methods for earthquakes and explosion discrimination, $J$. Geophys. Res., 90, 3009-3020.

Tarantola, A., Inverse Problem Theory, Elsevier Science, Amsterdam, The Netherlands, 1987.

Taylor, S., (1996). Analysis of high-frequency Pg/Lg ratios from NTS explosions and Western U.S. earthquakes, Bull. Seism. Soc. Am., 86, 1042-1053.

Taylor, S.R., and H.E. Hartse, (1998). A procedure for estimation of source and propagation amplitude corrections for regional seismic discriminants, J. Geophys. Res., 103, 2781-2789. 
Taylor, S.R., A.A. Velasco, H.E. Hartse, W. S. Phillips, W.R. Walter, and A.J. Rodgers (2002). Amplitude corrections for regional seismic discriminants, Pure. App. Geophys. (in press).

Taylor, S.R., X. Yang, and W. S. Phillips, Bayesian Lg attenuation tomography applied to eastern Asia, Los Alamos National Laboratory, Los Alamos, NM, LAUR-01-6601, 2001.

Walter, W. R., K. Mayeda, and H. J. Patton, (1995). Phase and spectral ratio discrimination between NTS earthquakes and explosions Part 1: Empirical observations, Bull. Seism. Soc. Am., 85., 1050-1067. 